\title{
A influência da violência familiar e entre pares na prática do bullying por adolescentes escolares
}

\author{
The influence of family and peer violence in the practice \\ of bullying among school adolescents
}

Georgia Rodrigues Reis e Silva (https://orcid.org/0000-0003-4486-2844) ${ }^{1}$ Maria Luiza Carvalho de Lima (https://orcid.org/0000-0002-1354-9890) ${ }^{2}$ Raquel Moura Lins Acioli (https://orcid.org/0000-0002-5907-0068) ${ }^{3}$

Alice Kelly Barreira (https://orcid.org/0000-0002-0351-9022) ${ }^{4}$
${ }^{1}$ Programa de Doutorado em Saúde Pública, Instituto Aggeu Magalhães (IAM), Fiocruz/PE. Av. Professor Moraes Rego s/n, Cidade Universitária. 50740465 Recife PE Brasil. georodrigues19@

yahoo.com.br

${ }^{2}$ Instituto Aggeu Magalhães (IAM), Fiocruz/PE. Recife

PE Brasil.

${ }^{3}$ Prefeitura do Recife. Recife PE Brasil.

${ }^{4}$ Departamento de Clínica e Odontologia Preventiva, Universidade Federal de Pernambuco (UFPE). Recife PE Brasil.

\begin{abstract}
The objective of this study was to estimate the prevalence of bullies and identify factors related to bullying among high school students in Recife. This is an analytical cross-sectional epidemiological study, composed of a probabilistic sample by clusters of 1,402 students aged 15 to 19 years old, enrolled in the second year of high school in both public and private schools in Recife. In this work the bullying will be approached according to the Bioecological Theory of Human Development of Bronfenbrenner. Data were collected by means of a self-administered questionnaire about bullying and associated factors. Data analysis was conducted by the Pearson chi-square test with statistical significance of 0.05 and 95\% Confidence Interval. To analyze associations, a multilevel modeling was employed and STATA version 12.0 software was used. The results showed a prevalence of $21.26 \%$ of bullying aggressors and the factors associated with the practice of bullying were violence suffered at school, violence between peers, as well as violence practiced by the mother and siblings. Hence, the results indicate that the violence suffered in the family and school context can have repercussions on the practice of bullying among adolescents.
\end{abstract}

Key words Bullying, Violence, Adolescent, School
Resumo $O$ objetivo deste estudo foi estimar a prevalência de agressores de bullying e identificar os fatores relacionados a este comportamento em adolescentes escolares do Recife. Trata-se de um estudo epidemiológico de corte transversal analítico, composto por uma amostra probabilística por conglomerados de 1.402 estudantes de 15 a 19 anos matriculados no segundo ano do ensino médio de escolas públicas e privadas do Recife. Neste trabalho, o bullying será abordado segundo a teoria bioecológica do desenvolvimento humano de Bronfenbrenner. A coleta de dados ocorreu por meio de um questionário autoaplicável com questões sobre bullying e fatores associados. A análise dos dados foi constituída pela aplicação do teste qui-quadrado de Pearson, considerando significância estatística de 0,05 e intervalo de confiança de 95\%. Para a análise das associações foi empregada a modelagem multinível e o software utilizado foi o STATA, versão 12.0. Os resultados mostraram prevalência de 21,26\% de agressores de bullying e os fatores associados à essa prática foram as violências sofridas na escola e entre pares, bem como a violência praticada pela mãe $e$ irmãos. Dessa forma, os resultados indicam que as violências sofridas no contexto familiar e escolar podem repercutir na prática do bullying entre adolescentes.

Palavras-chave Bullying, Violência, Adolescente, Escola 


\section{Introdução}

O bullying é um tipo de violência estudado principalmente no ambiente escolar e pode se manifestar de diferentes formas, a partir de comportamentos como agressões físicas e verbais ou até por ameaças, acusações injustas, roubo de dinheiro e pertences, difamações sutis e degradação de imagem social, adotados por um ou mais estudantes contra outro(s) com o objetivo de machucar, prejudicar ou humilhar, sem ter havido provocação por parte da vítima ${ }^{1-3}$. Ese fenômeno é caracterizado por três elementos essenciais: a repetição, a intencionalidade e a desequilíbrio de poder ${ }^{1,2,4,5}$.

A literatura revela que as diferentes manifestações de violência no âmbito escolar vêm comprometendo não só a saúde dos adolescentes, mas a qualidade da educação no contexto da escola pública brasileira ${ }^{6}$, já que esse comportamento entre os alunos tem prejudicado a realização das atividades escolares, o processo de aprendizagem, bem como ocasionado sentimento de insegurança na escola e, muitas vezes, abandono escolar? Também acarreta um processo de exclusão moral e social com repercussões imediatas e futuras na vida adulta dos envolvidos ${ }^{3}$, como o desenvolvimento de distúrbios mentais e ideação suicida ${ }^{8}$.

$\mathrm{O}$ bullying ocorre em processos interacionais dinâmicos e pode envolver os indivíduos de diversas maneiras, de forma que esses assumam papéis diferenciados, como: agressores, vítimas, vítimas/agressores ou testemunhas ${ }^{3,9}$. O papel de agressor se apresenta como o indivíduo que pratica a violência contra outro(s), supostamente mais fraco ${ }^{3}$. Os agressores geralmente são alunos do sexo masculino, mais velhos, que praticam mais sexo e mais exercício físico, apresentam comportamentos antissociais, faltam aulas, relatam solidão e insônia, possuem maior tendência a apresentar comportamentos de risco, como o uso de álcool e drogas ${ }^{1,4,6,10,11}$. Comumente fazem parte de pequenos ou grandes grupos liderados por um deles, que domina outros, e esses se subordinam à liderança por medo ou por satisfação de pertencerem ao grupo dominante ${ }^{1,4}$. No Brasil, alguns estudos que avaliaram adolescentes agressores de bullying verificaram prevalência entre 8,4 e $19,8 \% \%^{6,12,13}$.

Apesar de haver várias pesquisas brasileiras sobre a temática ${ }^{12-16}$, existem muitas lacunas no conhecimento que precisam ser abordadas. Estudos sobre fatores de proteção e intervenções efetivas ainda estão em estágios relativamente iniciais, e também há uma necessidade urgente de pesquisas mais rigorosamente avaliadas e relatadas em países de baixa e média renda ${ }^{17,18}$. Além disso, grande parte dos estudos analisam prioritariamente as vítimas e determinados fatores associados, sem utilizar um modelo explicativo e sem contemplar os diferentes contextos e interações que permeiam tal situação.

Diante da complexidade do fenômeno, neste trabalho o bullying será abordado segundo a teoria bioecológica do desenvolvimento humano de Bronfenbrenner ${ }^{19}$. Essa teoria apresenta quatro componentes multidirecionais interrelacionados, designados como modelo PPCT: os processos proximais; as características pessoais; o contexto; e o tempo. Neste estudo serão investigados os processos proximais, foco principal da teoria, que são as interações que a pessoa estabelece com seus pares, objetos e símbolos nos diversos contextos vivenciados em um determinado tempo histórico, nos quais o ser humano se apresenta como agente ativo de seu desenvolvimento, capaz de transformar o ambiente e transformar-se $e^{19,20}$.

A teoria de Bronfenbrenner é indicada para o estudo da violência por levar em consideração os quatro componentes PPCT no desenvolvimento individual como uma evolução complexa, com aquisição de competências e habilidades pessoais que irão conduzir e direcionar o comportamento nas mais diversas situações, como na escolha de uma atitude pacífica ou violenta. Nessa perspectiva, deve ser considerada a relevância de um estudo fundamentado em um modelo conceitual explicativo com o objetivo de estimar a prevalência de agressores de bullying e identificar os fatores de processo associados ao comportamento dos adolescentes agressores em escolas públicas e privadas do Recife.

\section{Método}

Trata-se de um estudo epidemiológico de corte transversal, de cunho analítico, que permitiu verificar a magnitude do evento e identificar as variáveis envolvidas no papel de agressor de bullying.

\section{Participantes}

A população do estudo foi composta por adolescentes, com idades entre 15 e 19 anos, matriculados no segundo ano do ensino médio de escolas públicas e particulares da cidade do Recife no período diurno. A faixa etária estendida para além do padrão para essa série foi incluída 
devido ao grande número de alunos com defasagem escolar.

\section{Amostra}

Para a estimativa da amostra, foram considerados os dados fornecidos pela Secretaria Estadual de Educação de Pernambuco. Consideraramse os seguintes parâmetros para estimar o total de alunos, segundo o número de alunos das escolas públicas e privadas matriculados em 2013: prevalência de bullying entre os alunos $=30 \%{ }^{7}$, erro amostral $=5 \%$, e efeito de desenho $=2$. A estimativa encontrada foi de 625 alunos de escola pública e 625 alunos de escola privada. Admitindo $20 \%$ de perda, perfaz um total de 1.563 alunos.

A amostragem foi realizada por conglomerados em dois estágios para atingir a amostra estimada. No primeiro, os parâmetros para estimar o número de escolas segundo esfera de governo foram: prevalência de bullying estimada em 95\% das escolas, erro amostral $=10 \%$, e efeito de desenho $=1$. A estimativa encontrada foi de 32 escolas, 16 públicas e 16 privadas. Admitindo $20 \%$ de perda, perfaz um total de 40 escolas distribuídas igualmente nos dois extratos. Assim, as escolas foram sorteadas, proporcionalmente à quantidade de alunos matriculados no segundo ano do ensino médio em cada escola, com intervalo sistemático.

No segundo estágio, as turmas foram sorteadas aleatoriamente dentro de cada escola. A fim de alcançar o quantitativo esperado de participantes, em cada escola foi aplicado em torno de 40 questionários, o que implicou a participação de uma ou duas turmas por escola, dependendo do tamanho das mesmas.

\section{Instrumento}

O instrumento de coleta consistiu em um questionário autoaplicável incluindo escalas e questões sobre o bullying e fatores associados. No entanto, neste artigo foram analisadas questões sobre agressor de bullying e as variáveis categorizadas segundo a dimensão "processo" da teoria bioecológica do desenvolvimento humano ${ }^{19}$. O envolvimento de bullying na condição de agressor foi definido a partir da questão produzida, baseada no conceito de Olweus ${ }^{1}$, e na Escala Califórnia de Vitimização do Bullying ${ }^{21,22}$, conforme descritas a seguir: "Dentre os(As) SeUs(suas) COLEGAS, EXISTE ALGUM(A) QUE VOCÊ CONSIDERA INFERIOR A VOCÊ? Se EXISTE, RESPONDA À PRÓXIMA QUESTÃo PENSANDO APENAS NESSA PESSOA. Alguma vez você já fez coisas de propósito, para magoar esse(a) seu(sua) colega, como: A) Zoar, xingar ou colocar apelidos? B) Espalhar boatos ou fofocas, ignorar ou deixar de fora do seu grupo? C) Empurrar, agredir fisicamente, ameaçar ou danificar coisas dele(a)?" Tendo para cada item as seguintes opções de respostas: 0 . Nunca; 1 . Apenas uma vez no mês passado; 2. Duas ou três vezes no mês passado; 3 . Apenas uma vez durante esta semana; e 4. Várias vezes durante esta semana. Sendo considerado agressor o indivíduo que assinalou um dos itens 2, 3 ou 4 em qualquer uma das alternativas.

As variáveis independentes analisadas neste estudo são: ter sofrido violência da mãe e do pai, ter sofrido violência na comunidade e na escola, sofrer e praticar violência física entre namorados, violência entre pares, violência entre irmãos, monitoramento dos pais e responsáveis, ter sofrido discriminação pelos vizinhos, ter sofrido discriminação pelos familiares, ter sofrido agressão sexual e conexão com a escola.

Uma vez que não existe uma escala composta por uma única variável que avalie a "conexão com a escola", tal avaliação ocorreu por meio da School Connectedness Scale ${ }^{23,24}$, adaptada pelos autores, composta por cinco itens relacionados ao adolescente separadamente: sentir-se próximo às pessoas da escola; sentir-se parte da escola; sentir-se feliz em estar na escola; sentir-se seguro na escola; os professores tratam os alunos com justiça. As variáveis independentes e instrumentos utilizados nesta pesquisa estão descritos no Quadro 1.

\section{Procedimentos}

A coleta de dados ocorreu no período de agosto a novembro de 2014, por meio da aplicação do questionário aos alunos presentes nas turmas sorteadas. Foram aplicados por dois pesquisadores, previamente treinados, que forneceram uma breve explicação sobre o preenchimento do questionário e permaneceram na sala de aula durante todo o processo, que teve duração média de 60 minutos em cada turma.

\section{Análise dos dados}

A análise dos dados foi constituída inicialmente de uma análise descritiva das variáveis, seguida da aplicação do teste qui-quadrado de Pearson com significância estatística de 0,05 e intervalo de confiança de $95 \%$.

Para a análise de associações, foi empregado a modelagem multinível para controle do efeito do 
Quadro 1. Descrição das variáveis, operacionalização e categorização das variáveis estudadas.

\begin{tabular}{|c|c|c|}
\hline Variável & Operacionalização & Categorização \\
\hline \multicolumn{3}{|l|}{ Dependente } \\
\hline Agressor de bullying & $\begin{array}{l}\text { Questão elaborada a partir da Escala Califórnia } \\
\text { de Vitimização do Bullying }{ }^{21} \text {, adaptada e } \\
\text { validada }^{22}\end{array}$ & Agressor \\
\hline \multicolumn{3}{|l|}{ Independentes } \\
\hline \multicolumn{3}{|c|}{ Variáveis de processo } \\
\hline $\begin{array}{l}\text { Sentir-se próximo as pessoas } \\
\text { da escola }\end{array}$ & \multirow[t]{5}{*}{$\begin{array}{l}\text { Questões elaboradas a partir do questionário } \\
\text { longitudinal Add Health }\end{array}$} & 1.Sim; 2. Não \\
\hline Sentir-se parte da escola & & 1.Sim; 2. Não \\
\hline $\begin{array}{l}\text { Sentir-se feliz em estar na } \\
\text { escola }\end{array}$ & & 1.Sim; 2. Não \\
\hline $\begin{array}{l}\text { Professores tratam alunos com } \\
\text { justiça }\end{array}$ & & 1.Sim; 2. Não \\
\hline Sentir-se seguro na escola & & 1.Sim; 2. Não \\
\hline Ter sofrido violência da mãe & \multirow[t]{2}{*}{$\begin{array}{l}\text { Escala Tática de Conflitos (CTS) validada para } \\
\text { população brasileira por Moraes, Hasselmann e } \\
\text { Reichenheim }(2002)^{25}\end{array}$} & $\begin{array}{l}\text { 1. Nunca sofreu; } 2 \text {. Sofreu } \\
\text { poucas vezes; } 3 \text {. Sofreu } \\
\text { muitas vezes }\end{array}$ \\
\hline Ter sofrido violência do pai & & $\begin{array}{l}\text { 1. Nunca sofreu; } 2 \text {. Sofreu } \\
\text { poucas vezes; } 3 \text {. Sofreu } \\
\text { muitas vezes }\end{array}$ \\
\hline $\begin{array}{l}\text { Ter sofrido violência na } \\
\text { comunidade }\end{array}$ & \multirow[t]{2}{*}{ ILANUD/ONU ${ }^{26}$} & 1. Sim; 2. Não \\
\hline Ter sofrido violência na escola & & 1.Sim; 2. Não \\
\hline $\begin{array}{l}\text { Sofrer e praticar violência } \\
\text { física entre namorados }\end{array}$ & $\begin{array}{l}\text { Conflict in Adolescent Dating Relationships } \\
\text { Inventory - CADRI }{ }^{27} \text {, adaptada por Minayo, } \\
\text { Assis e Njaine }(2011)^{28}\end{array}$ & 1. Sim; 2. Não \\
\hline Violência entre pares & $\begin{array}{l}\text { Escala Califórnia de Vitimização do Bullying }{ }^{21} \text {, } \\
\text { adaptada e validada }^{22}\end{array}$ & 1. Sim; 2. Não \\
\hline Violência entre irmãos & Questão elaborada & 1. Sim; 2. Não \\
\hline $\begin{array}{l}\text { Monitoramento dos pais e } \\
\text { responsáveis }\end{array}$ & Questão elaborada & $\begin{array}{l}\text { 1. Monitorado; } \\
\text { 2. Não é monitorado }\end{array}$ \\
\hline $\begin{array}{l}\text { Ter sofrido discriminação } \\
\text { pelos vizinhos }\end{array}$ & Questão elaborada & $\begin{array}{l}\text { 1. Nunca; } 2 \text {. Poucas vezes; } \\
\text { 3. Muitas vezes }\end{array}$ \\
\hline $\begin{array}{l}\text { Ter sofrido discriminação } \\
\text { pelos familiares }\end{array}$ & Questão elaborada & $\begin{array}{l}\text { 1. Nunca; } 2 \text {. Poucas vezes; } \\
\text { 3. Muitas vezes }\end{array}$ \\
\hline Ter sofrido agressão sexual & Questão elaborada & 1. Sim; 2. Não \\
\hline
\end{tabular}

Fonte: Elaborado pelos autores.

conglomerado e ponderada pelo tipo de escola. A técnica multivariada utilizada foi a stepwise do tipo forward, considerando como critério de entrada no modelo significância estatística de $10 \%$. Foram testadas as hipóteses de concomitância e considerada a variável no modelo segundo a plausibilidade causal. O software utilizado na análise foi o STATA, versão 12.0

\section{Questões éticas}

Esta pesquisa faz parte de um estudo maior, intitulado de "Bullying entre adolescentes escola- res: uma abordagem bioecológica", que foi aprovado pelo Comitê de Ética em Pesquisa do Instituto Aggeu Magalhães, de acordo com as normas do Conselho Nacional de Saúde sobre pesquisas envolvendo seres humanos.

Antes do início da coleta, foram solicitadas autorizações institucionais no âmbito da educação e assinaturas dos Termos de Consentimento Livre e Esclarecido e Termos de Assentimento Livre e Esclarecido. Os questionários foram respondidos de maneira anônima e os resultados publicados garantirão anonimato dos participantes. 


\section{Resultados}

A amostra foi composta por 1.402 estudantes, a maioria do sexo feminino (52,4\%), com média de idade de 16,4 anos. Quanto ao tipo de escola, $56,4 \%$ cursavam as escolas públicas e $43,6 \%$ as privadas. A frequência de bullying na condição de agressor foi de 298 casos, correspondendo a uma prevalência de 21,26\% (IC 95\%: 17,84\% a 24,39\%).

A partir da análise univariada foi observada, com relação às interações pessoais permeadas por violência, uma associação significativa com a prática de bullying, seja com a violência sofrida na escola $(\mathrm{OR}=2,46(1,82-3,34), \mathrm{p}<0,001)$, na comunidade $(\mathrm{OR}=2,05(1,62-2,58), \mathrm{p}<0,001)$ ou relacionada à violência entre namorados, seja ela sofrida $(O R=1,76(1,21-2,55), p=0,003)$ ou praticada $(\mathrm{OR}=1,83(1,19-2,81), \mathrm{p}=0,006) . \mathrm{A}$ violência sofrida pelo adolescente e praticada pela mãe, seja ela verbal $(\mathrm{OR}=1,98(1,32-2,97)$, $\mathrm{p}=0,001)$ ou física $(\mathrm{OR}=2,15(1,66-2,78), \mathrm{p}<$ $0,001)$ mostrou-se associada à prática do bullying pelo jovem, assim como a violência física sofrida pelo jovem, sendo o pai o agressor $(\mathrm{OR}=1,71$ (1,30-2,25), $\mathrm{p}<0,001)$. Além disso, a violência entre irmãos, nas formas física $(\mathrm{OR}=2,19(1,63$ $2,94), \mathrm{p}<0,001)$ e psicológica $(\mathrm{OR}=2,14(1,57-$ 2,93), $\mathrm{p}<0,001)$ mostrou-se associada a uma maior possibilidade de praticar o bullying, assim como a condição de familiares terem o costume de perseguir o jovem $(\mathrm{OR}=1,80(1,16-2,79)$, $\mathrm{p}$ $=0,009)$. A associação da prática do bullying e a condição de o jovem ter sofrido agressão sexual $(\mathrm{OR}=1,88(1,05-3,35), \mathrm{p}=0,031)$ também foi estatisticamente significante, apontando uma maior chance de prática de bullying entre aqueles que foram vítimas alguma vez na vida. Ainda no que se refere à violência entre pares, os alunos que a relataram apresentaram 3,40 mais chances de serem agressores de bullying $(\mathrm{p}<0,001)$.

Também foi verificada associação das atitudes discriminatórias cometidas pelos vizinhos e a prática de bullying. A ocorrência de atos discriminatórios no que diz respeito à orientação sexual $(\mathrm{OR}=2,89(1,60-5,24), \mathrm{p}=0,000)$ e aparência física $(\mathrm{OR}=1,75(1,28-2,40), \mathrm{p}=0,000)$ foram significativamente relacionados ao aumento da chance de prática de bullying, e entre os familiares os atos discriminatórios relativos à cor da pele $(\mathrm{OR}=2,02(1,19-3,42), \mathrm{p}=0,009)$ orientação sexual $(\mathrm{OR}=2,74(1,31-5,75), \mathrm{p}=0,007)$ e aparência física $(\mathrm{OR}=1,81(1,15-2,84), \mathrm{p}=0,010)$ também foram associados a uma maior chance de prática de bullying.
Em relação à conexão com a escola, verificouse uma associação estatisticamente significante com a condição de agressor no que diz respeito aos adolescentes "se sentirem parte da escola" (OR = 0,74 (0,56-0,98), $\mathrm{p}=0,038)$, "se sentirem feliz em estar na escola" (OR $=0,57(0,43-0,74)$, $\mathrm{p}<0,001$ ), "se sentirem seguro na escola" (OR $=0,54(0,41-0,73), \mathrm{p}<0,001)$ ou quando consideraram que os "professores tratam com justiça" $(\mathrm{OR}=0,54(0,39-0,76), \mathrm{p}<0,001)$.

Por fim, o monitoramento dos pais e responsáveis (poucas vezes ou nenhum monitoramento) também foi associado à prática de bullying $(\mathrm{OR}=2,20(1,57-3,08) \mathrm{p}<0,001)$, como está descrito da Tabela 1.

A partir da análise do modelo multivariado (Tabela 2), verificou-se que a violência sofrida na escola $(\mathrm{p}<0,001)$, assim como a experiência de violência entre pares $(\mathrm{p}<0,001)$, aumentou a chance de ser agressor de bullying.

A violência física especificamente cometida pela mãe $(\mathrm{p}<0,001)$ mostrou-se associada à prática de bullying. Ainda em relação à violência intrafamiliar, observou-se uma associação estatisticamente significante com a violência física ( $p$ $=0,006)$ e verbal $(p=0,030)$ entre irmãos.

Apesar de não se manter no modelo final, o monitoramento dos pais e responsáveis mostrou-se limítrofe na associação com a prática do bullying, com $\mathrm{p}=0,058$.

Não houve associação significativa ao final da análise multivariada com as demais variáveis de processo estudadas.

\section{Discussão}

A prevalência encontrada na presente pesquisa corrobora os achados publicados em $2014^{29} \mathrm{em}$ estudo realizado no Brasil em escolas da rede pública e privada nas capitais brasileiras e no Distrito Federal, no qual foi verificada prevalência de agressores de 20,8\%. É um fato relevante, pois aponta para uma semelhança entre a realidade das escolas locais e as nacionais do ponto vista de alunos que afirmam praticar tal comportamento. Outras pesquisas realizadas no $\mathrm{Brasil}^{30,31}$ e na Nigéria $^{32}$ encontraram frequências ainda mais elevadas de agressores de bullying, obtendo 48,9\%, $54,7 \%$ e $64,9 \%$ de agressores, respectivamente. Em contrapartida, estudo realizado com adolescentes em 40 países $^{33}$ evidenciou prevalência de prática de bullying de $10,7 \%$, porém com grande variação nas taxas entre os países. Tais dados podem refletir as diferenças sociais, culturais e 
Tabela 1. Análise univariada dos fatores de processo associados ao bullying na condição de agressor de bullying.

\begin{tabular}{|c|c|c|c|c|}
\hline \multirow[b]{2}{*}{ Variáveis relacionadas a processo } & \multicolumn{4}{|c|}{ Agressor de bullying $(\mathrm{n}=1.402)$} \\
\hline & $\begin{array}{c}\text { Sim } \\
\text { N (\%) }\end{array}$ & $\begin{array}{c}\text { Não } \\
\text { N (\%) }\end{array}$ & $\mathrm{OR}^{\mathrm{a}}(\mathrm{IC}$ 95\%) & p-valor ${ }^{b}$ \\
\hline \multicolumn{5}{|l|}{ Ter sofrido violência na escola } \\
\hline Não & $75(13,20 \%)$ & $493(86,80 \%)$ & Referência & \\
\hline Sim & $212(27,4 \%)$ & $561(72,57 \%)$ & $2,46(1,82-3,34)$ & $<0,001$ \\
\hline \multicolumn{5}{|c|}{ Ter sofrido violência na sua comunidade } \\
\hline Não & $108(15,6 \%)$ & $584(84,39 \%)$ & Referência & \\
\hline Sim & $174(27,3 \%)$ & $462(72,64 \%)$ & $2,05(1,62-2,58)$ & $<0,001$ \\
\hline \multicolumn{5}{|l|}{ Violência entre pares } \\
\hline Não & $113(13,1 \%)$ & $748(86,8 \%)$ & Referência & \\
\hline Sim & $184(34,0 \%)$ & $356(65,93 \%)$ & $3,40(2,68-4,31)$ & $<0,001$ \\
\hline \multicolumn{5}{|l|}{ Violência entre namorados } \\
\hline \multicolumn{5}{|l|}{ Violência sofrida } \\
\hline Não & $46(18,78 \%)$ & $199(81,22 \%)$ & Referência & \\
\hline Sim & $92(29,39 \%)$ & $221(70,61 \%)$ & $1,76(1,21-2,55)$ & 0,003 \\
\hline Nunca namorou & $155(18,7 \%)$ & $674(81,30 \%)$ & $0,96(0,70-1,33)$ & 0,846 \\
\hline \multicolumn{5}{|l|}{ Violência praticada } \\
\hline Não & $42(17,72 \%)$ & $195(82,28 \%)$ & Referência & \\
\hline Sim & $94(28,92 \%)$ & $231(71,08 \%)$ & $1,83(1,19-2,81)$ & 0,006 \\
\hline Nunca namorou & $157(19,0 \%)$ & $667(80,95 \%)$ & $1,07(0,74-1,54)$ & 0,712 \\
\hline \multicolumn{5}{|l|}{ Ter sofrido violência da mãe } \\
\hline \multicolumn{5}{|l|}{ Violência verbal } \\
\hline Não & $38(13,15 \%)$ & $251(86,85 \%)$ & Referência & - \\
\hline Sim & $259(23,3 \%)$ & $852(76,69 \%)$ & $1,98(1,32-2,97)$ & 0,001 \\
\hline \multicolumn{5}{|l|}{ Violência física } \\
\hline Não & $110(15,2 \%)$ & $615(84,83 \%)$ & Referência & - \\
\hline Sim & $188(27,8 \%)$ & $488(72,2 \%)$ & $2,15(1,66-2,78)$ & $<0,001$ \\
\hline \multicolumn{5}{|l|}{ Ter sofrido violência do pai } \\
\hline \multicolumn{5}{|l|}{ Violência verbal } \\
\hline Não & $141(19,1 \%)$ & $595(80,84 \%)$ & Referência & - \\
\hline Sim & $149(22,8 \%)$ & $503(77,15 \%)$ & $1,24(0,96-1,61)$ & 0,093 \\
\hline \multicolumn{5}{|l|}{ Violência física } \\
\hline Não & $218(19,4 \%)$ & $905(80,59 \%)$ & Referência & - \\
\hline Sim & $80(28,8 \%)$ & $197(71,12 \%)$ & $1,71(1,30-2,25)$ & $<0,001$ \\
\hline \multicolumn{5}{|c|}{ Monitoramento dos pais ou responsáveis } \\
\hline Sempre/muitas vezes & $233(19,5 \%)$ & $964(80,53 \%)$ & Referência & - \\
\hline Poucas/nunca & $58(34,32 \%)$ & $111(64,68 \%)$ & $2,20(1,57-3,08)$ & $<0,001$ \\
\hline
\end{tabular}

políticas existentes em cada nação ou também as medidas preventivas eficazes no enfrentamento ao fenômeno.

Essa questão é reforçada pelos resultados que mostram que os sentimentos de segurança, felicidade, sentir-se parte da escola e considerar os professores justos são fatores de proteção à prática de bullying, embora não tenham permanecido na análise final.

Em relação às variáveis de processo, observase que houve associação com a violência sofrida na escola, a violência física sofrida pela mãe e pelos irmãos, bem como da violência entre pares. Fato preocupante, tendo em vista o impacto que esse tipo de conduta pode trazer para os que sofrem as agressões como modelo de reprodução do comportamento vivenciado. Considerando que os principais locais de socialização e preparação para a vida adulta desses adolescentes são a escola e a casa, esses adolescentes têm experienciado as diversas formas de violência, desde bullying até a violência familiar, nos seus 
Tabela 1. Análise univariada dos fatores de processo associados ao bullying na condição de agressor de bullying.

\begin{tabular}{|c|c|c|c|c|}
\hline \multirow[b]{2}{*}{ Variáveis relacionadas a processo } & \multicolumn{4}{|c|}{ Agressor de bullying $(\mathrm{n}=1.402)$} \\
\hline & $\begin{array}{c}\text { Sim } \\
\mathrm{N}(\%)\end{array}$ & $\begin{array}{c}\text { Não } \\
\text { N (\%) }\end{array}$ & $\mathrm{OR}^{\mathrm{a}}(\mathrm{IC}$ 95\%) & p-valor \\
\hline \multicolumn{5}{|l|}{ Violência entre irmãos } \\
\hline \multicolumn{5}{|l|}{ Violência física } \\
\hline Não & $118(15,8 \%)$ & $626(84,8 \%)$ & Referência & - \\
\hline Sim & $114(29,1 \%)$ & $277(70,84 \%)$ & $2,19(1,63-2,94)$ & $<0,001$ \\
\hline \multicolumn{5}{|l|}{ Violência verbal } \\
\hline Não & $56(17,18 \%)$ & $270(82,82 \%)$ & Referência & - \\
\hline Sim & $175(21,6 \%)$ & $636(78,42 \%)$ & $1,30(0,97-1,75)$ & 0,072 \\
\hline \multicolumn{5}{|l|}{ Violência psicológica } \\
\hline Não & $133(16,4 \%)$ & $677(83,58 \%)$ & Referência & - \\
\hline Sim & $96(30,09 \%)$ & $223(69,91 \%)$ & $2,14(1,57-2,93)$ & $<0,001$ \\
\hline \multicolumn{5}{|l|}{ Familiares costumam perseguir } \\
\hline Poucas vezes/nunca & $260(20,3 \%)$ & $1017(79,6 \%)$ & Referência & - \\
\hline Sempre/muitas vezes & $36(31,58 \%)$ & $78(68,42 \%)$ & $1,80(1,16-2,79)$ & 0,009 \\
\hline \multicolumn{5}{|l|}{ Ter sofrido agressão sexual } \\
\hline Não & $270(20,8 \%)$ & $1025(79,1 \%)$ & Referência & - \\
\hline Sim & $14(33,3 \%)$ & $28(66,7 \%)$ & $1,88(1,05-3,35)$ & 0,031 \\
\hline \multicolumn{5}{|l|}{ Ter sofrido discriminação pelos vizinhos } \\
\hline Pela cor da pele & $17(24,29 \%)$ & $53(75,71 \%)$ & $1,26(0,67-2,37)$ & 0,467 \\
\hline Condição social & $30(28,30 \%)$ & $76(71,70 \%)$ & $1,58(0,98-2,55)$ & 0,056 \\
\hline Orientação sexual & $19(39,58 \%)$ & $29(60,42 \%)$ & $2,89(1,60-5,24)$ & 0,000 \\
\hline Aparência física & $64(29,22 \%)$ & $155(70,7 \%)$ & $1,75(1,28-2,40)$ & 0,000 \\
\hline \multicolumn{5}{|c|}{ Ter sofrido discriminação pelos familiares } \\
\hline Pela cor da pele & $13(36,1 \%)$ & $23(63,9 \%)$ & $2,02(1,19-3,42)$ & 0,009 \\
\hline Condição social & $14(26,9 \%)$ & $38(73,1 \%)$ & $1,42(0,80-2,53)$ & 0,201 \\
\hline Orientação sexual & $18(40,0 \%)$ & $27(60,0 \%)$ & $2,74(1,31-5,75)$ & 0,007 \\
\hline Aparência física & $46(30,87 \%)$ & $103(69,1 \%)$ & $1,81(1,15-2,84)$ & 0,010 \\
\hline \multicolumn{5}{|l|}{ Conexão com a escola } \\
\hline Sentir-se próximo as pessoas & $219(20,3 \%)$ & $858(79,6 \%)$ & $0,76(0,58-1,01)$ & 0,062 \\
\hline Sentir-se parte da escola & $198(19,9 \%)$ & $797(80,1 \%)$ & $0,74(0,56-0,98)$ & 0,038 \\
\hline Sentir-se feliz em estar na escola & $185(18,4 \%)$ & $820(81,6 \%)$ & $0,57(0,43-0,74)$ & $<0,001$ \\
\hline Professores tratam com justiça & $174(18,0 \%)$ & $790(81,9 \%)$ & $0,54(0,39-0,76)$ & $<0,001$ \\
\hline Sentir-se seguro na escola & $185(18,7 \%)$ & $803(81,2 \%)$ & $0,54(0,41-0,73)$ & $<0,001$ \\
\hline
\end{tabular}

Fonte: Elaborado pelos autores.

diversos espaços de convivência ${ }^{34}$. Todavia, esses deveriam ser ambientes em que o adolescente se sentisse acolhido e protegido, ao mesmo tempo em que pudesse expandir seus conhecimentos e aprender a ser solidário, a ter consciência dos direitos, deveres e responsabilidades, e a lidar com os problemas com independência, ou seja, a ser um cidadão.

No que diz respeito ao estudante ter sofrido violência física pela mãe, essa conduta pode estar relacionada à tentativa de inibir um comportamento inadequado do filho ou puni-lo, pois conforme destaca Andrade ${ }^{35}$, o comportamento agressivo tem origem no modelo educativo familiar, a partir da agressividade e violência cometida pelos pais para punição dos filhos, de modo a influenciar no comportamento social desses ${ }^{14}$. Zottis e colaboradores ${ }^{36}$ também verificaram que a prática de bullying apresentou associação com a disciplina parental autoritária e punitiva, sobretudo o castigo severo praticado especificamente pela mãe. Além disso, chamam atenção quanto ao uso de castigo corporal por parte dos pais, pois dessa forma ensinam a seus filhos que 
Tabela 2. Análise multivariada dos fatores de processo associados ao bullying na condição de agressor de bullying.

\begin{tabular}{|c|c|c|}
\hline \multirow[t]{2}{*}{$\begin{array}{c}\text { Variáveis relacionadas } \\
\text { a processo }\end{array}$} & \multicolumn{2}{|l|}{$\begin{array}{l}\text { Agressor de } \\
\text { bullying }\end{array}$} \\
\hline & $\mathrm{OR}^{\mathrm{a}}(\mathrm{IC}$ 95\%) & p-valor ${ }^{b}$ \\
\hline \multicolumn{3}{|l|}{$\begin{array}{l}\text { Violência sofrida na } \\
\text { escola }\end{array}$} \\
\hline Não & Referência & \\
\hline Sim & $1,76(1,28-2,41)$ & $<0,001$ \\
\hline \multicolumn{3}{|l|}{$\begin{array}{l}\text { Violência física sofrida } \\
\text { pela mãe }\end{array}$} \\
\hline Não & Referência & \\
\hline Sim & $1,74(1,35-2,23)$ & $<0,001$ \\
\hline \multicolumn{3}{|l|}{ Violência entre pares } \\
\hline Não & Referência & \\
\hline Sim & $2,61(2,00-3,40)$ & $<0,001$ \\
\hline \multicolumn{3}{|l|}{$\begin{array}{l}\text { Violência física sofrida } \\
\text { pelos irmãos }\end{array}$} \\
\hline Não & Referência & \\
\hline Sim & $1,67(1,15-2,41)$ & 0,006 \\
\hline \multicolumn{3}{|l|}{$\begin{array}{l}\text { Violência verbal sofrida } \\
\text { pelos irmãos }\end{array}$} \\
\hline Não & Referência & \\
\hline Sim & $0,66(0,46-0,96)$ & 0,030 \\
\hline
\end{tabular}

Fonte: Elaborado pelos autores.

bater é uma das maneiras de lidar com conflitos interpessoais.

Outro fato importante a ser destacado é que Malta et al., em $2010^{37}$, já haviam publicado pesquisa nacional realizada nas capitais e no Distrito Federal, com escolares de escola públicas e privada, que a maior prevalência de violência física no âmbito doméstico foi registrada na cidade do Recife, com 11,7\% (IC 95\%: 10,3\%-13,0\%). Assim, revela que esse tipo de violência acontece há algum tempo, sendo frequente nessa faixa etária e nesse contexto.

A violência entre pares, que é caracterizada como ao menos uma experiência de vitimização ou ato de violência de qualquer frequência entre pares, mas sem a presença de desequilíbrio de poder $^{1,21}$, também se revelou associada à prática de bullying. Nesse caso, presume-se que o estudante envolvido em uma situação eventual de violência entre colegas, seja na situação de vítima ou de agressor, mesmo que não de forma intencional, esteja mais propenso a reproduzir alguma agressão, passando a agir de intencionalmente diante de alguém visivelmente mais fraco, mais tímido ou mais novo.

Estudo recente empreendido no Brasil também verificou que a maioria dos agressores sofre violência física familiar ${ }^{11}$. Corroborando tais informações, pesquisas apontam que o envolvimento de adolescente com a violência se deve a diversos fatores, entre os quais a própria fase da adolescência, o consumo abusivo de substâncias, os conflitos e a violência na família, a exposição à violência e a situações de violência ou conflito conjugal adolescentes ${ }^{38,39}$.

Além dos fatores citados, verificou-se que o pouco ou nenhum monitoramento dos pais mostrou-se limítrofe na associação ao bullying no papel de agressor. Dado semelhante ao apresentando em pesquisa que mostrou que ser agressor se associou inversamente à supervisão familiar ${ }^{11}$. Já Ayas ${ }^{40}$ verificou que estudantes que tinham pais com atitudes negligentes sofriam mais bullying do que aqueles com pais com atitudes permissivas e democráticas. Apesar deste trabalho não analisar o bullying no papel de agressor, observase como é importante o monitoramento e participação dos pais na vida dos filhos, tendo em vista que esse vem sendo associado tanto a casos de vítimas quanto de agressores.

Nesse sentido, existem fortes evidências de que o comportamento do agressor de bullying está moldado pelas interações e experiências de violência vivenciadas ao longo da vida, as quais Bronfenbrenner classifica como a dimensão processo.

Embora a presente pesquisa tenha abordado apenas a violência interpessoal que ocorre nos contextos familiar e escolar, precisamos considerar o ambiente de violência generalizada ao qual estão expostas as pessoas que vivem em capitais brasileiras. A teoria de Bronfenbrenner mostra que o desenvolvimento humano ocorre em um sistema de multicamadas que se estende desde a relação de nível micro entre pais e filhos até instituições e culturas de nível macro e se desdobra ao longo do tempo. Para as pessoas prosperarem como indivíduos, seres sociais e cidadãos, precisam de condições que lhes permitam florescer fisicamente e desenvolver relacionamentos e ações cada vez mais inclusivos e expansivos, como participantes sociais e cívicos ${ }^{41}$.

Esta pesquisa apresenta algumas limitações com relação aos dados. Por serem baseados apenas em autorrelatos, há a possibilidade de o estudante falsear a resposta ou apresentar o viés de 
memória. Outra limitação foi a inclusão somente de adolescentes que estavam frequentando a escola e presentes em sala de aula no momento da aplicação do questionário, o que pode ter ocasionado algum viés nos resultados, pois o absenteísmo e a evasão escolar estão relacionados ao problema estudado. No entanto, não inviabiliza o trabalho, pois foi realizado o cálculo amostral considerando tal acontecimento. Além disso, o presente estudo oferece uma percepção pontual da realidade, não permitindo estabelecer uma relação causal, tendo em vista ser um estudo transversal.

Conclui-se que existe uma alta prevalência de prática de bullying na população estudada, e os fatores associados são as violências sofridas na escola, a violência entre pares e a violência praticada pela mãe e pelos irmãos. Os dados ressaltam a importância de compreender as relações e interações dos agressores de bullying com as pessoas em seus diversos espaços, a fim de planejar ações específicas.

Dessa forma, nota-se que as estratégias de prevenção e combate ao bullying devem envolver alunos, pais e/ou responsáveis, profissionais de educação e da saúde. Tendo em vista que essa prática pode ser consequência da violência sofrida na escola, por pares e por familiares no domicílio, as medidas junto aos alunos agressores não podem ser apenas punitivas, e sim de apoio e escuta especializada para que possam lidar com seus sentimentos diante da violência sofrida e refletir acerca dos motivos que os levaram a cometer tais atos. É importante a conscientização dos adolescentes agressores quanto às consequências desses comportamentos nas suas vidas e nas dos que sofrem bullying, como o desenvolvimento de doenças psicossomáticas e problemas sociais, emocionais e acadêmicos ${ }^{3,16}$.

No contexto escolar, os profissionais da educação precisam estar preparados para enfrentar essas situações, a ponto de distinguir a violência e as brincadeiras próprias da idade, bem como promover discussões e atividades sobre o tema e intervir ao presenciar determinados comportamentos, favorecendo uma cultura de paz. Os profissionais de saúde têm papel importante no que se refere às ações de combate à violência escolar, não só por meio de intervenções com o programa de saúde na escola, mas identificando nas unidades de saúde adolescentes vítimas de violência e notificando as entidades responsáveis. Pais e/ ou responsáveis precisam compreender que os comportamentos agressivos não são adequados e por isso devem ser evitados dentro da família. A cultura da paz precisa estar presente em todos os ambientes os quais estes adolescentes estão inseridos, inclusive no seu domicílio. Portanto, aproximar os pais da escola é imprescindível para o enfrentamento do bullying escolar.

Apesar de existirem vários estudos brasileiros sobre o bullying, a maioria é relativa à vitimização e poucos usaram um olhar mais integrador quanto ao conjunto dessas variáveis, além de não apresentarem um modelo conceitual explicativo para tal fenômeno. Dessa forma, ratifica-se a importância deste trabalho no sentido de contribuir para a elaboração de ações e programas que visem a promoção à prevenção e à intervenção a esse tipo de comportamento.

\section{Colaboradores}

GRR Silva foi responsável pela tabulação, análise dos dados e pela escrita do manuscrito. MLC Lima foi responsável pela concepção do projeto, supervisão da coleta de dados e revisão do manuscrito. AK Barreira foi responsável pela construção do projeto, supervisão da coleta de dados e revisão crítica do manuscrito. RML Acioli foi responsável pela construção do projeto e coleta dos dados. 


\section{Financiamento}

Este trabalho foi financiado pelo Programa PAPES VI/FIOCRUZ/CNPq.

\section{Referências}

1. Olweus D. Bullying at school: what we know and what we can do. Cambridge: Blackwell; 1993.

2. Gladden RM, Vivolo-Kantor AM, Hamburger ME, Lumpkin CD. Bullying surveillance among youths: uniform definitions for public health and recommended data elements - Version 1.0. Atlanta: National Center for Injury Prevention and Control, Centers for Disease Control and Prevention and U.S. Department of Education; 2014.

3. Zequinão MA, Medeiros P, Pereira B, Cardoso FL. Bullying escolar: um fenômeno multifacetado. Educ Pesqui 2016; 42(1):181-98.

4. Berger KS. Update on bullying at school: science forgotten? Developmental Review 2007; 27(1):90-126.

5. Bandeira CM, Hutz CS. Bullying: prevalência, implicações e diferenças entre os gêneros. Psicol Esc Educ 2012; 19(1):35-44.

6. Mello FCM, Silva JL, Oliveira WA, Prado RR, Malta DC, Silva MAI. A prática de bullying entre escolares brasileiros e fatores associados, Pesquisa Nacional de Saúde do Escolar 2015. Cien Saude Colet 2017; 22(9):2939-2948.

7. Malta DC, Silva MAI, Mello FCM, Monteiro RA, Sardinha LMV, Crespo C, Carvalho MGO, Silva MMA, Porto DL. Bullying in Brazilian schools: results from the National School-based Health Survey (PeNSE), 2009. Cien Saude Colet 2010; 15(Suppl. 2):3065-3076.

8. Sigurdson JF, Undheim AM, Wallander JL, Lydersen $\mathrm{S}$, Sund AM. The longitudinal association of being bullied and gender with suicide ideations, self-harm, and suicide attempts from adolescence to young adulthood: a cohort study. Suicide Life Threat Behav 2018; 48(2):169-182.

9. Santos MM, Perkoski IR, Kienen N. Bullying: atitudes, consequências e medidas preventivas na percepção de professores e alunos do ensino fundamental. Temas Psicol 2015; 23(4):1017-1033.

10. Liang H, Flisher AJ, Lombard CJ. Bullying, Violence, and risk behavior in South African school students. Child Abuse Negl 2007; 31(2):161-171.

11. Oliveira WA, Silva MAI, Silva JL, Mello FCM, Prado RR, Malta DC. Associations between the practice of bullying and individual and contextual variables from the aggressors' perspective. J Pediatr 2016; 92(1):3239.

12. Marcolino EC, Cavalcanti AL, Padilha WWN, Miranda FAN, Clementino FS. Bullying: prevalência e fatores associados à vitimização e à agressão no cotidiano escolar. Texto Contexto Enferm 2018; 27(1): e5500016.

13. Alcantara SC, González-Carrasco M, Montserrat C, Casas F, Viñas-Poch F, Abreu DP. Violência entre pares, clima escolar e contextos de desenvolvimento: suas implicações no bem-estar. Cien Saude Colet 2019; 24(2):509-522.

14. Weimer WR, Moreira EC. Violência e bullying: manifestações e consequências nas aulas de educação física escolar. Rev Bras Cienc Esporte 2014; 36(1):257-274.

15. Monteiro RP, Medeiros ED, Pimentel CE, Soares AKS, Medeiros HA, Gouveia, VV. Valores humanos e bullying: idade e sexo moderam essa relação? Temas em Psicologia 2017; 25(3):1317-1328. 
16. Malta DC, Mello FCM, Prado RRD, Sá ACMGN, Marinho F, Pinto IV, Silva MMAD, Silva MAI. Prevalência de bullying e fatores associados em escolares brasileiros, 2015. Cien Saude Colet 2019; 24(4):1359-1368.

17. Sivaraman B, Nye E, Bowes L. School-based anti-bullying interventions for adolescents in low- and middle-income countries: a systematic review. Aggression and Violent Behavior 2019; 45:154-162.

18. Zych I, Farrington DP, Ttofi MM. Protective factors against bullying and cyberbullying: a systematic review of meta-analyses. Aggression and Violent Behavior 2019; 45:4-19.

19. Bronfenbrenner $U$. The bioecological theory of human development. In: Bronfenbrenner $U$, editor. Making humans beigns human: bioecological perspectives on human development. Thousand Oaks: Sage Publications; 2005. p. 3-15.

20. Martins E, Szymanski H. A abordagem ecológica de Urie Bronfenbrenner em estudos com famílias. Estud Pesqui Psicol 2004; 4(1):12-25.

21. Felix ED, Sharkey JD, Green JG, Furlong MJ, Tanigawa D. Getting precise and pragmatic about the assessment of bullying: the development of the California Bullying Victimization Scale. Aggress Behav 2011; 37(3):234-247.

22. Soares AKS, Gouveia VV, Gouveia RSV, Fonsêca PN, Pimentel CE. Escala Califórnia de Vitimização do Bullying (ECVB): evidências de validade e consistência interna. Temas Psicol 2015; 23(2):481-491.

23. Tourangeau R, Shinn H. National longitudinal study of adolescent health: grand sample weight. Chapel Hill: University of North Carolina, National Opinion Research Center and Carolina Population Center; 1999.

24. You S, Furlong MJ, Felix E, Sharkey JD, Tanigawa D. Relations among school connectedness, hope, life satisfaction, and bully victimization. Psychology in the Schools 2008; 45(5):446-460.

25. Moraes CL, Hasselmann MH, Reichenheim ME. Adaptação transcultural para o português do instrumento "Revised Conflict Tactics Scales (CTS2)" utilizado para identificar violência entre casais. Cad Saude Publica 2002; 18(1):163-175.

26. Kahn T, Bermergui C, Yamada E, Cardoso FC, Fernandes F, Zacchi JM, Guimarães L, Hasselman ME Projeto de pesquisa: o dia a dia nas escolas (violências auto-assumidas). São Paulo: Instituto Latino Americano das Nações Unidas para a Prevenção do Delito e Tratamento do Delinquente (ILANUD)/Instituto Sou da Paz; 1999.

27. Wolfe DA, Scott K, Reitzel-Jaffe D. Development and validation of the Conflict in adolescent dating relationship inventory. Psychol Assess 2001; 13(2):277293.

28. Minayo MCS, Assis SG, Njaine K, organizadores. Amor e violência: um paradoxo das relações de namoro $e$ do 'ficar' entre jovens brasileiros. Rio de Janeiro: Fiocruz; 2011.

29. Malta DC, Prado RR, Dias AJR, Mello FCM, Silva MAI, Costa MR, Caiaffa WT. Bullying e fatores associados em adolescentes brasileiros: análise da Pesquisa Nacional de Saúde do Escolar (Pense 2012). Rev Bras de Epidemiol 2014; 17(Supl. 1):131-145.
30. Brito CC, Oliveira MT. Bullying and self-esteem in adolescents from public schools. J Pediatr (Rio J). 2013; 89(6):601-607

31. Bandeira CM, Hutz CS. Bullying: prevalence, implications and gender differences. Psicol Esc Educ 2012; 16:35-44.

32. Alex-Hart BA, Okagua J, Opara PI. Prevalence of bullying in secondary schools in Port Harcourt. Int $J$ Adolesc Med Health 2015; 27(4):391-396.

33. Craig W, Harel-Fisch Y, Fogel-Grinvald H, Dostaler S, Hetland J, Simons-Morton B, Molcho M, de Mato MG, Overpeck M, Due P, Pickett W, HBSC Violence \& Injuries Prevention Focus Group, HBSC Bullying Writing Group. A cross-national profile of bullying and victimization among adolescents in 40 countries. Int J Public Health 2009; 54(Suppl. 2):216-224.

34. Abramovay M. Escola e violência. Brasília: Unesco, 2002.

35. Andrade MP. Bullying: concepções dos atores envolvidos [monografia]. Bauru: Universidade Estadual Paulista, Faculdade de Ciências; 2007.

36. Zottis GA, Salum GA, Isolan LR, Manfro GG, Heldt E. Associations between child disciplinary practices and bullying behavior in adolescents. J Pediatr (Rio J) 2014; 90(4):408-414.

37. Malta DC, Souza ER, Silva MMA, Silva CS, Andreazzi MAR, Crespo C, Mascarenhas MDM, Porto DL, Figueroa ALG, Morais Neto OL, Penna GO. Vivência de violência entre escolares brasileiros: resultados da Pesquisa Nacional de Saúde do Escolar (PeNSE). Cien Saude Colet 2010; 15(Supl. 2):3053-3063.

38. Horta RL, Horta BL, Pinheiro RT, Krindges M. Comportamentos violentos de adolescentes e coabitação parento-filial. Rev Saude Publ 2011; 44(6):979-985.

39. Faria CS, Martins CBG. Violência entre adolescentes escolares: condições de vulnerabilidades. Rev Electrónica Trimestral de Enfermaría 2016; 42:171-184.

40. Ayas T. The effect of parental attitudes on bullying and victimizing levels of secondary school students. Procedia-Social and Behavioral Sciences 2012; 55:226231.

41. Adams TM. How chronic violence affects human development, social relations, and the practice of citizenship: a systemic framework for action. Washington, DC: Woodrow Wilson International Center for Scholars; 2017.

Artigo apresentado em 13/12/2018

Aprovado em 03/10/2019

Versão final apresentada em 05/10/2019

Editores-chefes: Romeu Gomes, Antônio Augusto Moura da Silva 
